\title{
Evaluation of clomiphene citrate therapy on fertility success rate in infertile patients with ovulatory factors in Sarem hospital 1394-1396
}

\section{ARTICLE INFO}

Article Type

Review of article

Authors

Abootaleb Saremi ${ }^{1}$, iD MD

Anahita Nazempour ${ }^{2}$, MD*

${ }^{1}$ Sarem Fertility \& Infertility Research Center (SAFIR) \& Sarem Cell Research Center (SCRC), Sarem Women's Hospital, Iran University of Medical Sciences (IUMS), Tehran, Iran.

2 Sarem Fertility \& Infertility Research Center (SAFIR), Sarem Women's Hospital, Iran University of Medical Sciences (IUMS), Tehran, Iran.

\section{*Corresponding Author}

Address: Sarem Women Hospital, Basij Square, Phase 3, EkbatanTown, Tehran, Iran. Postal code:1396956111 Phone: +98 (21) 44670888 Fax: +98 (21) 44670432 dr.Nazempour@sarem.org

\section{Article History}

Received: September 05, 2020 Accepted: September 20, 2020 e Published: May 06, 2021

\section{ABSTRACT}

Aims: Ovarian factor is one of the most common causes of infertility in couples and various treatment methods have been adopted to treat it. Clomiphene drug is one of the safest, least expensive and most effective methods in these people. The drug is used with or without gonadotropins and IUI (Intra uterine insemination) method in infertile and subfertile couples with ovarian factors and health in the hypothalamic-pituitary-ovarian axis. The aim of this study was to evaluate the success rate of this drug in infertile women with ovarian factor that leads to a positive result. Positive results include pregnancies with a confirmed $\beta$ hCG test, vaginal ultrasound, and a pregnancy sac seen inside the uterine cavity. Therefore, the results of this study can be effective and useful in the physician's decision to continue treatment.

Methods: This study is a retrospective cross-sectional study that the results of treatment with clomiphene with or without IUI in 785 patients referred to the IVF clinic of Sarem subspecialty hospital with ovarian factor during 2015 to 1396 has been reviewed. Hormones level such as thyroid-stimulating hormone (TSH), follicle-stimulating hormone (FSH), luteinizing hormone (LH), estradiol (E2), prolactin (PRL), and antimalarial hormone $(\mathrm{AMH})$ were measured on the third day of menstruation and then clomiphene was prescribed. In all patients, spermogram test were performed twice, which was normal after the spermation process (Capacitation).

Findings: The results showed that there was no significant relationship between the type of menstruation and treatment protocol used for the patients $(\mathrm{P}=0.109)$. Also, there was no significant relationship between the presence of acne and hirsutism in patients and the type of treatment protocol $(\mathrm{P}=0.281$ and $\mathrm{P}=0.232$, respectively). In this study, there was no significant relationship between the type of clomiphene treatment or clomiphene with IUI and the levels of LH $(\mathrm{P}=0.783)$, FSH $(\mathrm{P}=0.516), \mathrm{AMH}(\mathrm{P}=0.679)$ and $\mathrm{PRL}(\mathrm{P}=0.345)$ and also there was no significant relationship between body mass index (BMI) with the type of patient treatment $(\mathrm{P}=0.584)$. Clomiphene treatment alone did not have a significant effect on the success of pregnancy outcomes, but in the clomiphene and IUI treatment protocol, there were 127 positive pregnancies, which $3 \%$ of them were ectopic pregnancies, and $5 \%$ of them was led to abortion. Finally, 124 cases resulted in the birth of a live baby, which based on the results of statistical analysis showed a significant relationship $(\mathrm{P}=0.036)$.

Conclusion: Clomiphene as a drug with low complication, low cost and available can be used as a suitable option in the treatment of infertile patients with ovarian factor in combination with IUI treatment method.

Keywords: Clomiphene, Ovulatory factors, Infertility, Subfertility, Protocol. 
يافته ها: نتايج حاصل از تحقيق نشان داد كه بين نوع قاعدكى و يروتكل

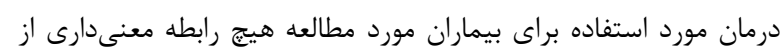

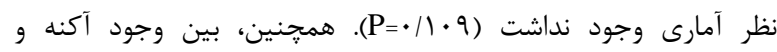

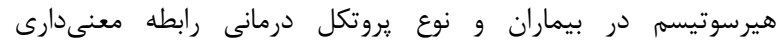

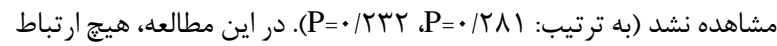

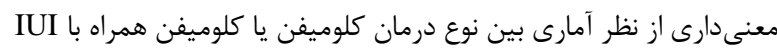

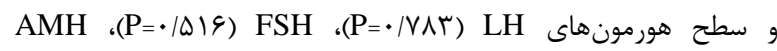

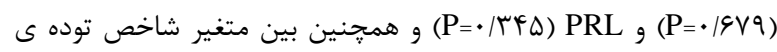

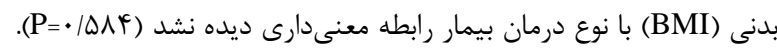

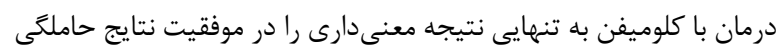

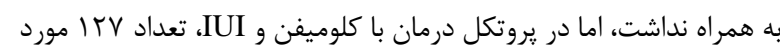

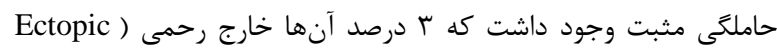

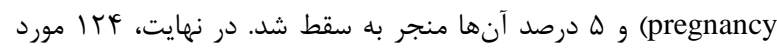

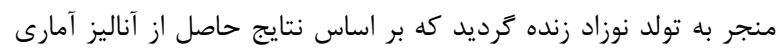

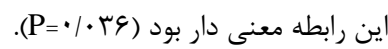

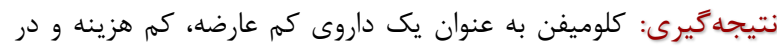

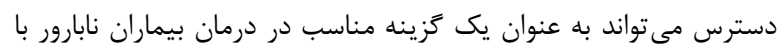

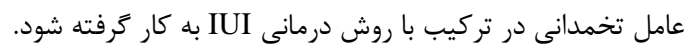

كليد وارهها: كلوميفن، فاكتور تخمدانى، نابارور، نيمه بارور، يروتكل.

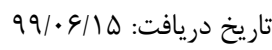

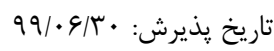

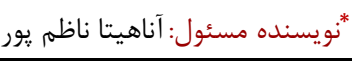

مقدمه

نابارورى و نيمه بارورى به زوجى اطلاق مى شود كه يس از اقدام به بارد دارى

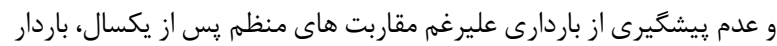

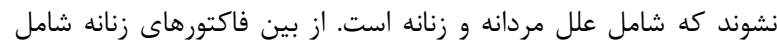

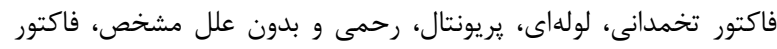

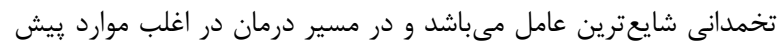

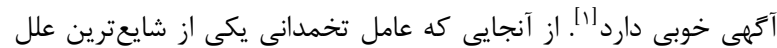

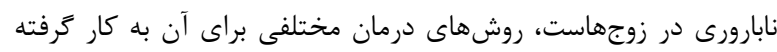

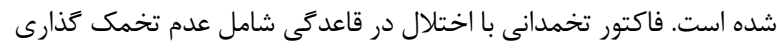

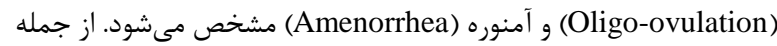

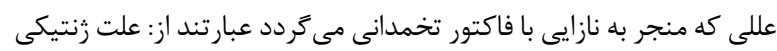

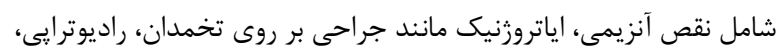

\section{بر رسى اثر ميزان موفقيت كلوميفن}

در بارورى خانمهاى نابارور با فاكتور تخمدانى در بيمارستان صارم از

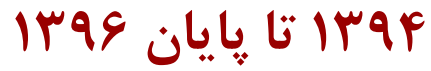

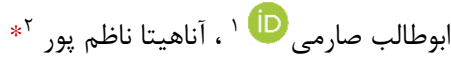

' مركز تحقيقات بارورى و نابارورى صارم، مركز تحقيقات سلولى و مولكولى

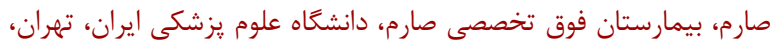
r مركز تحقيقات بارورى و نابارورى صارم، بيمارستان فوق تخصصى صارم، دانشگاه علوم يزشكى ايران، تهران، ايران.

جكيده

اهداف: عامل تخمدانى يكى از شايعترين علل نابارورى در زوجها بوده و

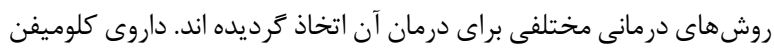

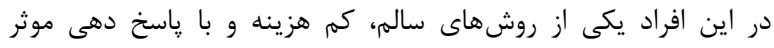

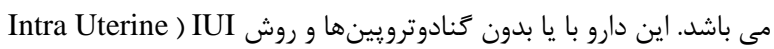
(Insemination

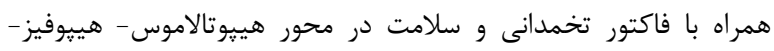

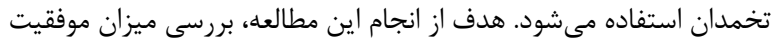

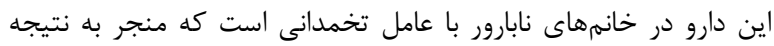

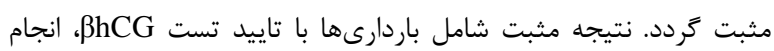

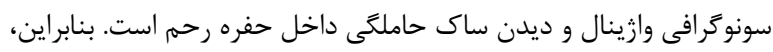

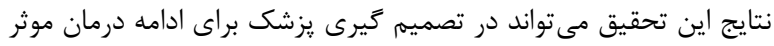

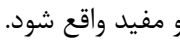
روش ها: اين بررسى به صورت يك مطالعه مق مقطعى كذشتهنكر است كه

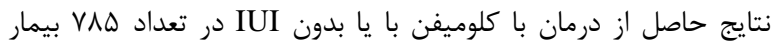

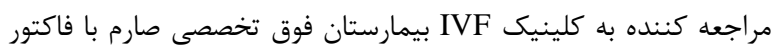

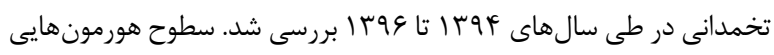

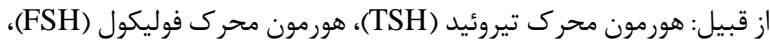

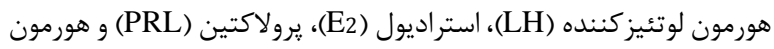

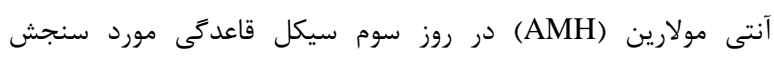

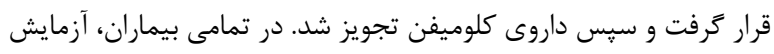

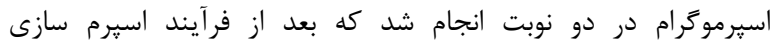
(Capacitation) 
بر سى اثر ميزان موفقيت كلوميفن در بارورى خانم هاى نابارور با فاكتور تخمدانى در بيمارستان صارم VV

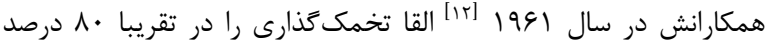

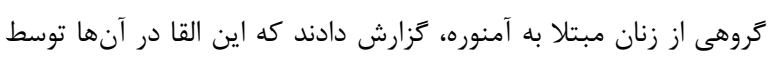

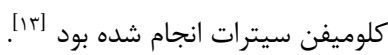

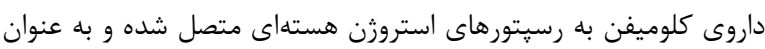

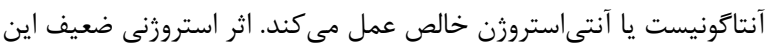

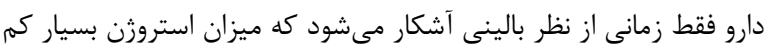

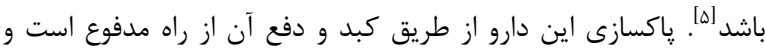

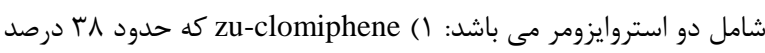

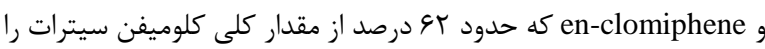
تشكيل مى دهد كه در اصل همان دو ايزومر فضايى متفاوت سيس و و ترانس

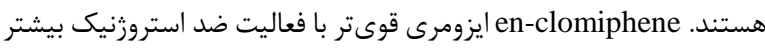

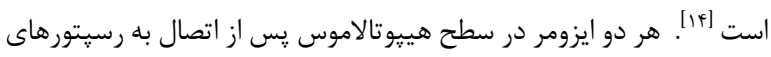

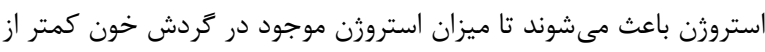

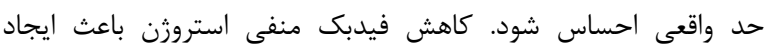

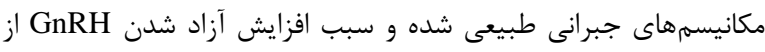

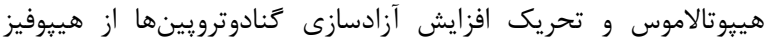

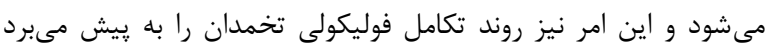

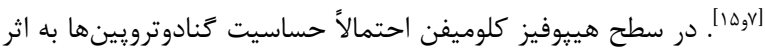

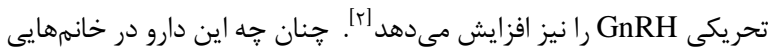

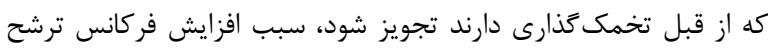

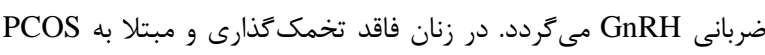

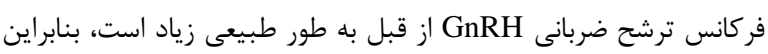

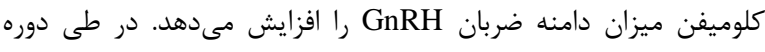

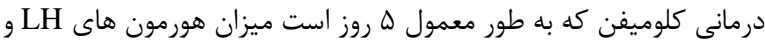

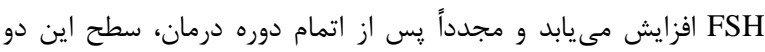

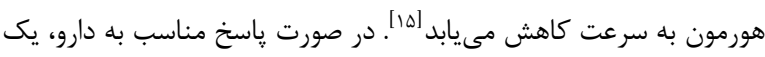

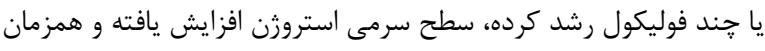

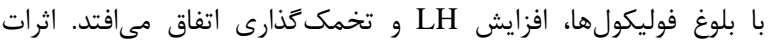

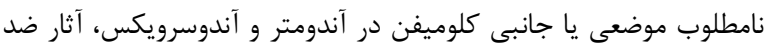

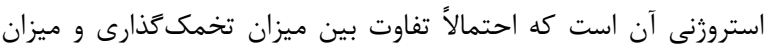

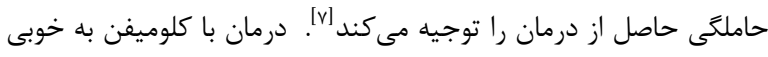

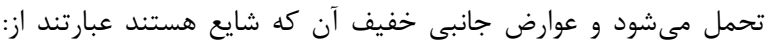

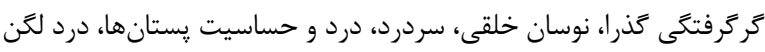

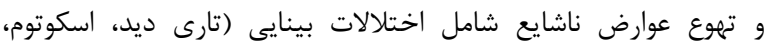

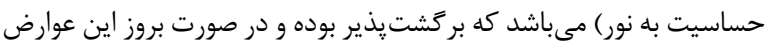

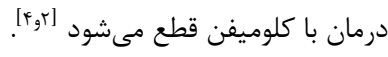

\section{مواد و روش ها}

اين مطالعه، يك مطالعه كذشتهنكر مقطعى است كه در يك بازه زمان زمانى سه

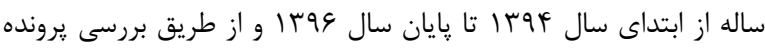

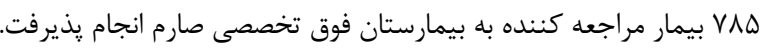

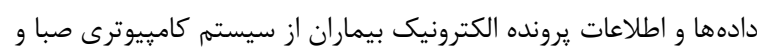

شيمى درمانى، بيمارىهاى زمينهاى مانند ديابت، سرطان، آندومتريوز،

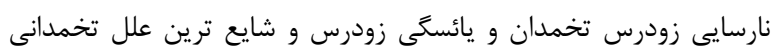

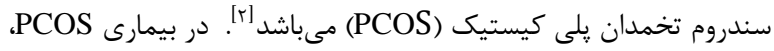

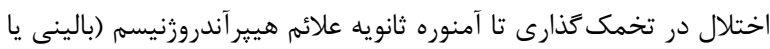

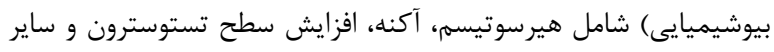

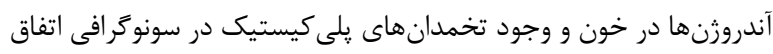

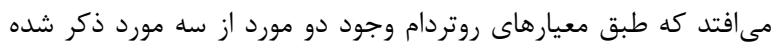

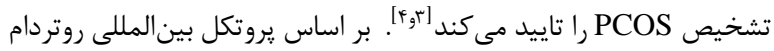

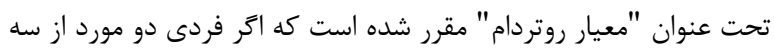

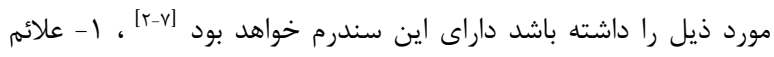

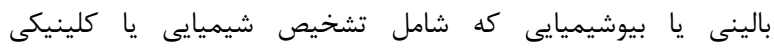

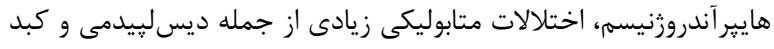

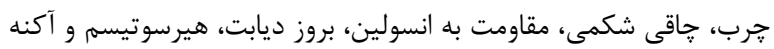

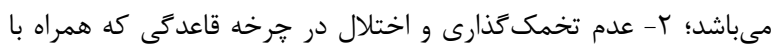
افزايش آندروزن و تاثيرى كه روى محور هييوفيز-هييوتالاموس مغز داشئ داشته

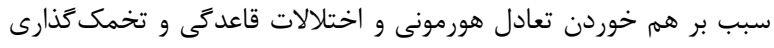

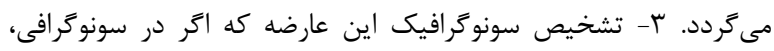

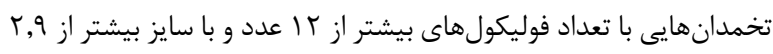

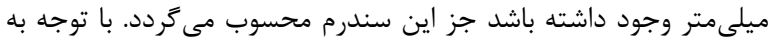

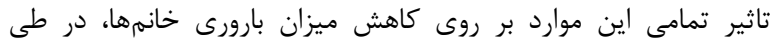

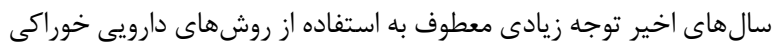

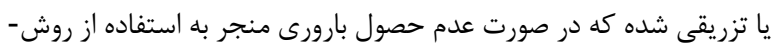

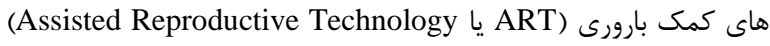

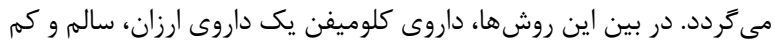

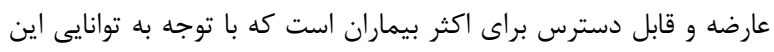

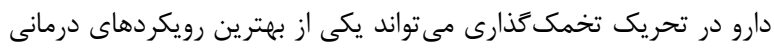

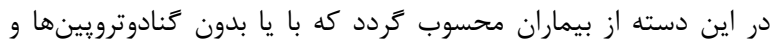

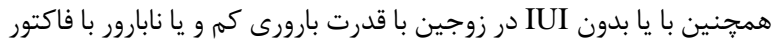

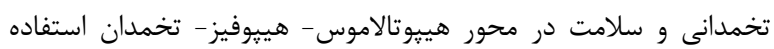

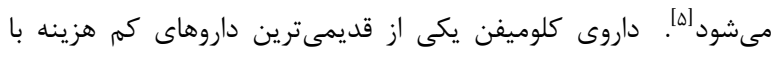

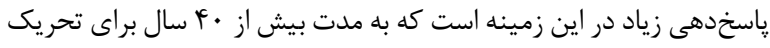

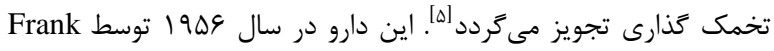

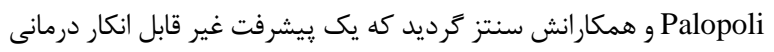

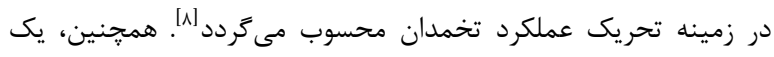

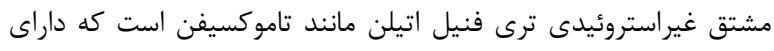

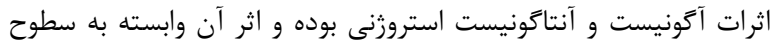

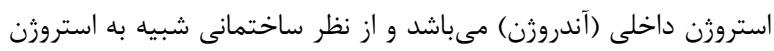

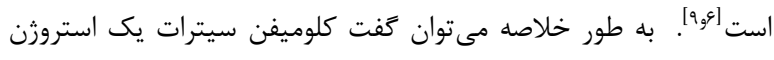

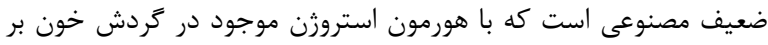

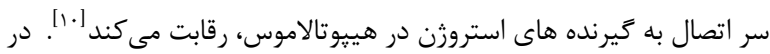

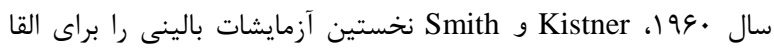

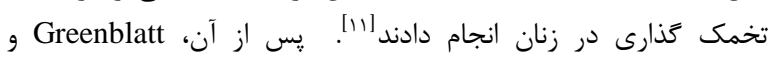




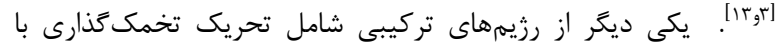

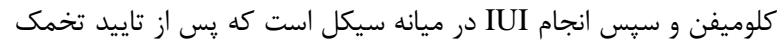

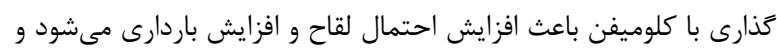

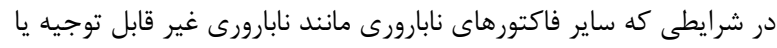

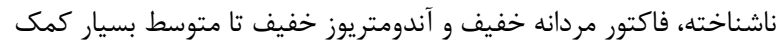

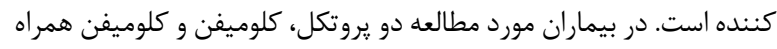

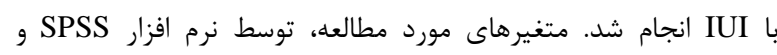

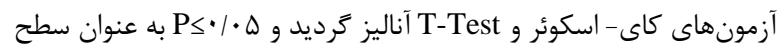
معنى دارى در نظر كرفته شد.

\section{يافته ها}

نتايج اين بررسى نشان داد كه ميانگين سنى خانمها ها ه تا هـ سال كه

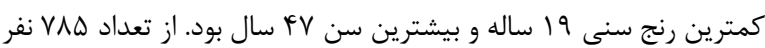

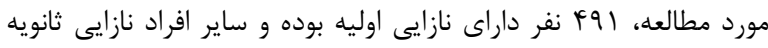

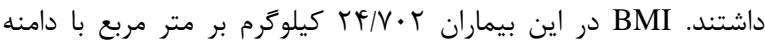

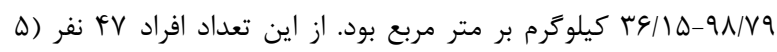

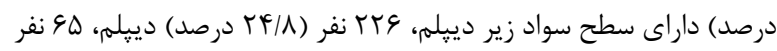

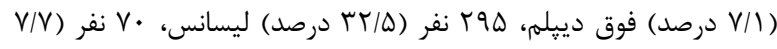

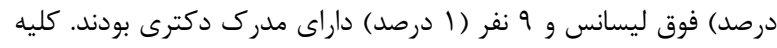

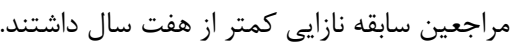

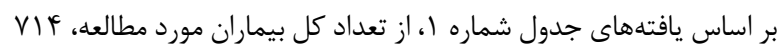

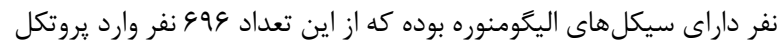

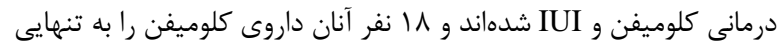

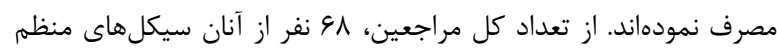

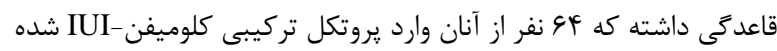

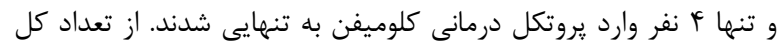

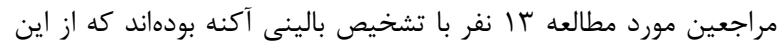

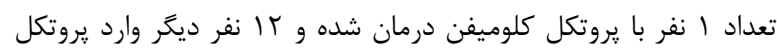

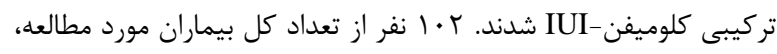

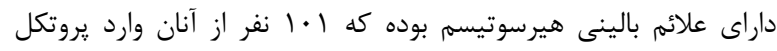

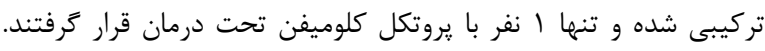

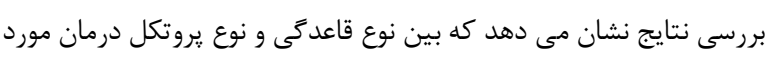

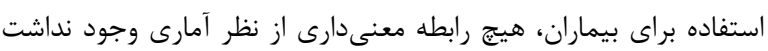

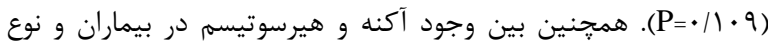

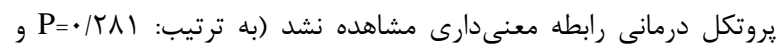
كبرو
سحر بيمارستان فوق تخصصى صارم دريافت شد. در اين سيستم براى هر

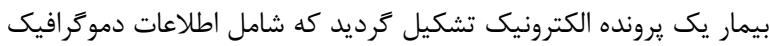
بيماران (سن، شغل، تحصيلات، قوميت ، وضعيت اشتغال، شرايط اجتماعى بردي

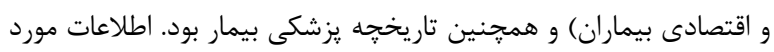

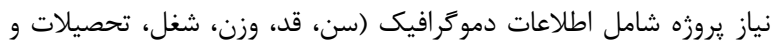

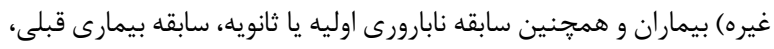

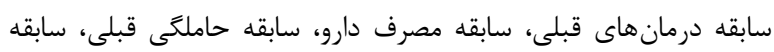

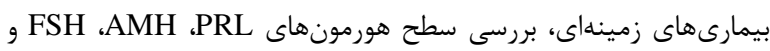
LH

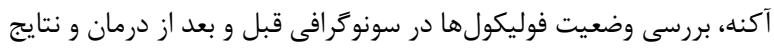

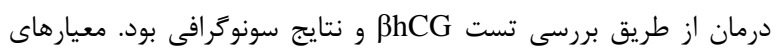

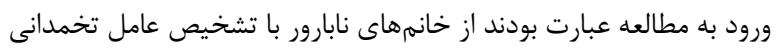

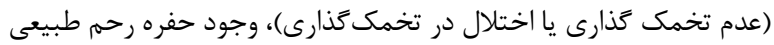

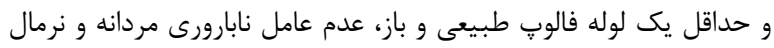

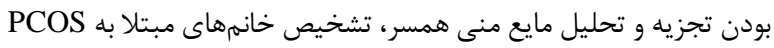

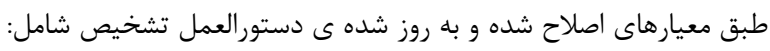

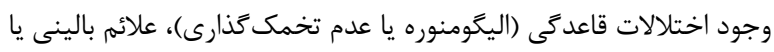

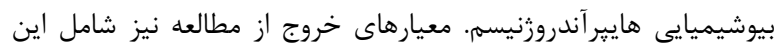

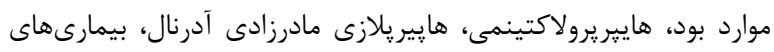

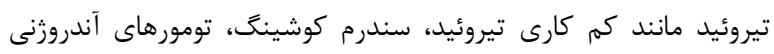

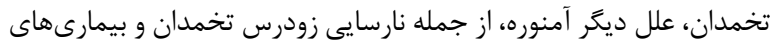

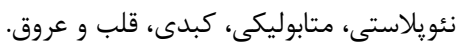

يروتكل مصرف داروى كلوميفن

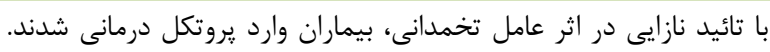

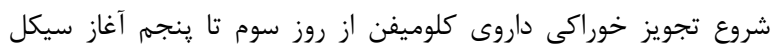

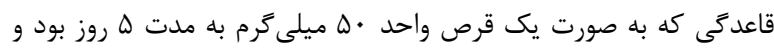

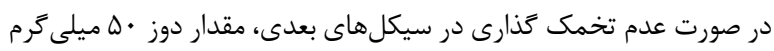

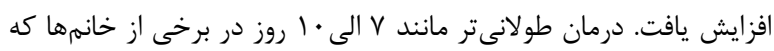

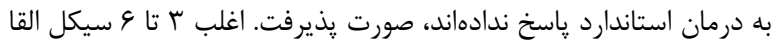

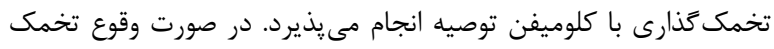

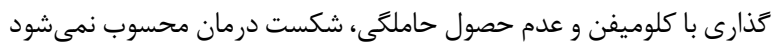

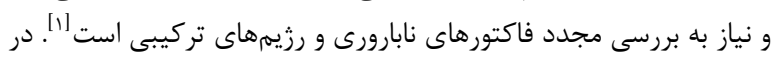

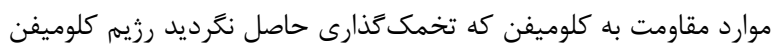

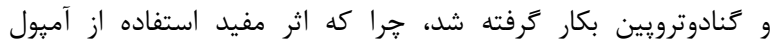

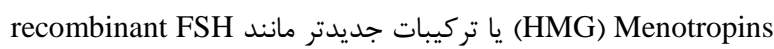

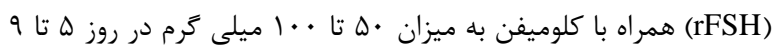

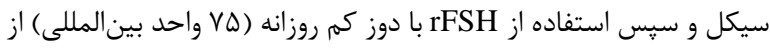

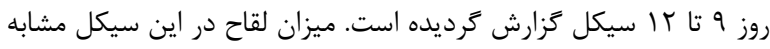

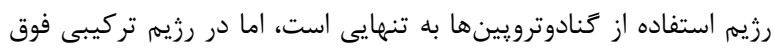

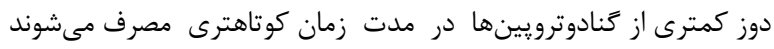


بحث عامل تخمدانى يكى از شايعترين علل نابارورى در ميان خانم هاى نابارور

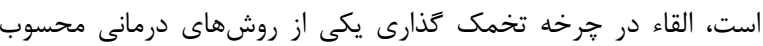

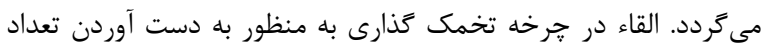

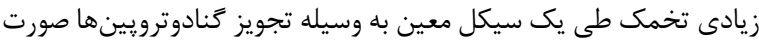

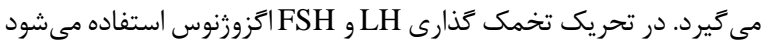

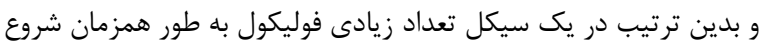

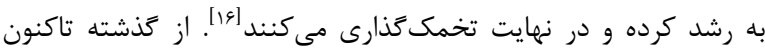

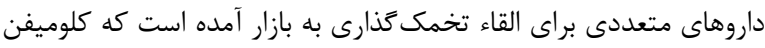

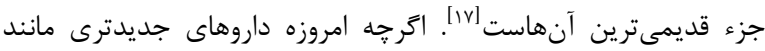

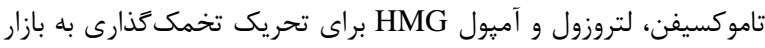

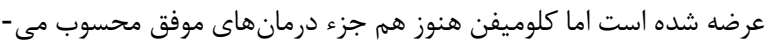

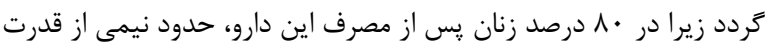

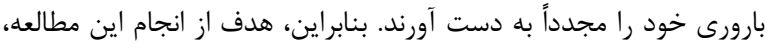

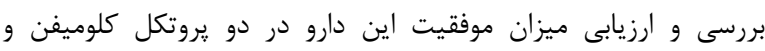

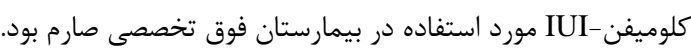

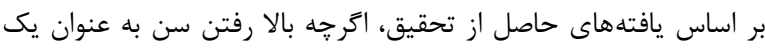

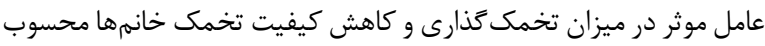

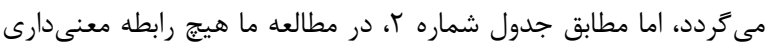
بين سن بيماران و نوع درمان (يروتكل كلوميفن و يروتكل كلوميفن- (IUI)

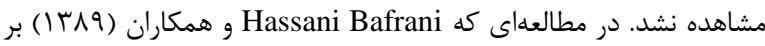

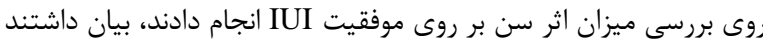

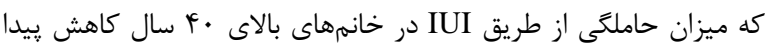

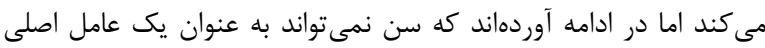

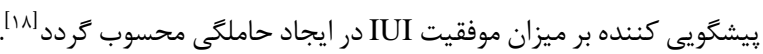

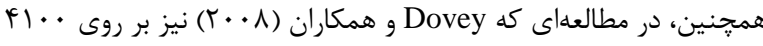

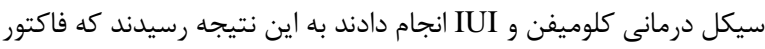

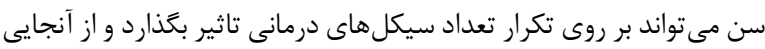

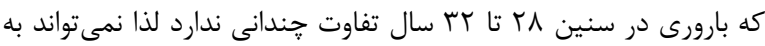

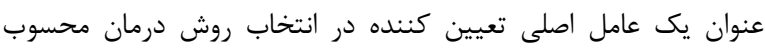

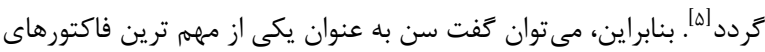

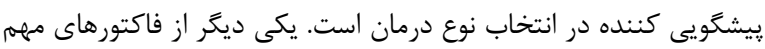

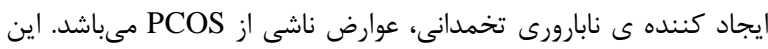

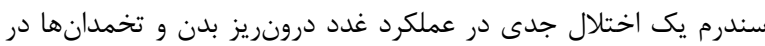

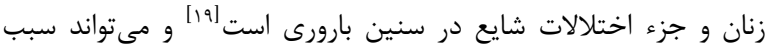

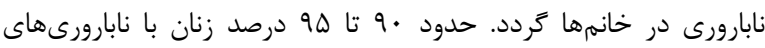

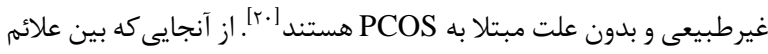

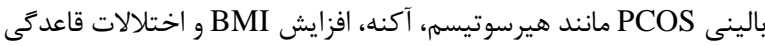

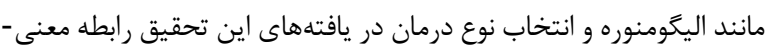

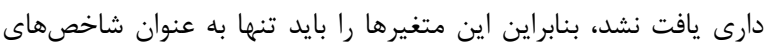

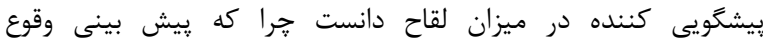

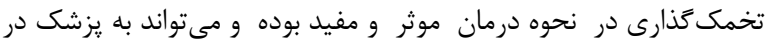

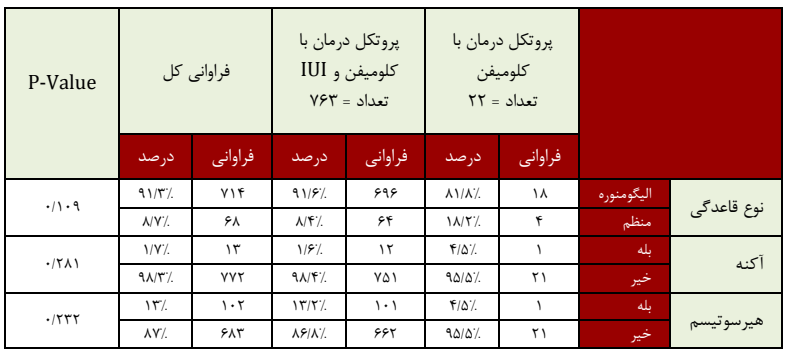

جدول ا: رابطه متغيرهاى مورد مطالعه و يروتكلهاى درمانى كلوميفن وكلوميفن-IUI

مطابق جدول شماره r، تاثير نوع درمان بر روى سن و ميزان هورمون هاى

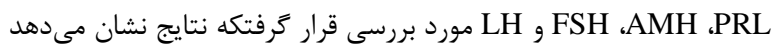

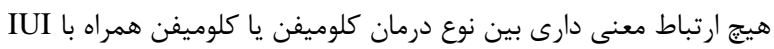
و سطح هورمونهاى

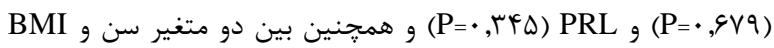

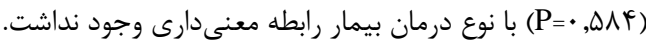

\begin{tabular}{|c|c|c|c|c|c|}
\hline \multirow[t]{2}{*}{ P-Value } & \multicolumn{2}{|c|}{ 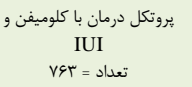 } & \multicolumn{2}{|c|}{ 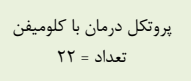 } & \\
\hline & انحراف از معيار & ميانكين & انحراف از معيار & ميانكين & \\
\hline - hrf & F/ar. & $r \Delta / T \Lambda$ & F/orq & $r F / 1 f$ & سن (سال) \\
\hline$\cdot / \Delta \Delta F$ & $f / . . F$ & TY/SA & F/NF & $r F / 09$ & (كيلوكرم بر متر مربع)BMI \\
\hline$\cdot$. NAT & firat & $\Delta / \Lambda T$ & $F / 190$ & Fiva & $(\mathrm{mIU} / \mathrm{L}) \mathrm{LH}$ \\
\hline.$/ 019$ & ri/99V & $9 / 111$ & $F / 4 \cdot 9$ & $\Delta / \Delta T$ & $(\mathrm{mIU} / \mathrm{L}) \mathrm{FSH}$ \\
\hline .1989 & r/arı & $r / 9 \gamma$ & FiTtF & ria9 & (ng/mL)AMH \\
\hline $.64 \Delta$ & $101 \cdot V V^{2}$ & velf. & $|v \Delta| r \cdot \Delta$ & 9T/9Y & (mIU/L)PRL \\
\hline
\end{tabular}

جدول rأ: بررسى سطح هورمونها در دو يروتكل درمانى كلوميفن وكلوميفن

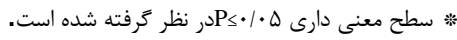

بر اساس نتايج جدول شماره ؟، درمان با كلوميفن به تنهايى نتيجه معنى-

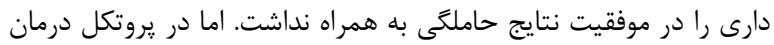

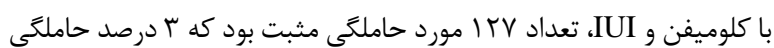

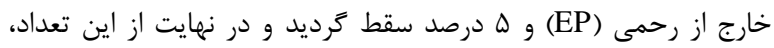

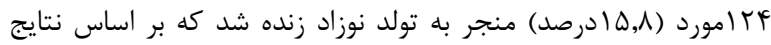

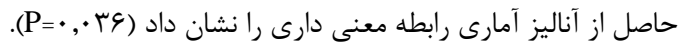

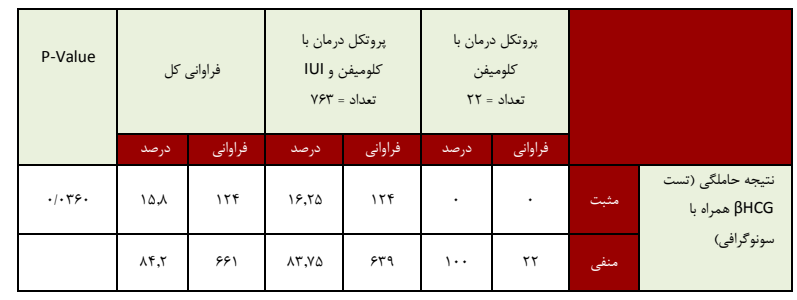

جدول rا پِيامد درمان با دو روش درمانى كلوميفن وكلوميفن-IUI دانشنامه صارم در طب بارورى 
بيش از حد تخمدان (OHSS) در مقايسه با ساير داروهاى مشابه نيز انجام

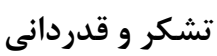

بدينوسيله از از همه همكاران محترم كلينيك نازايى بيمارستان فوق

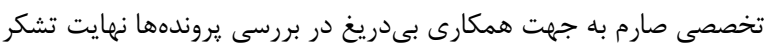

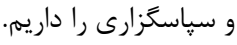

\section{تاييديه اخلاقى} اين مطالعه با كد SRI00021102F مورد تاييد كميته اخلاق موسسه تحقيقات صارم (SRI) قرار گرفت.

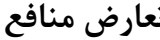 در اين مطالعه تعارض منافع وجود نداشت.}

\author{
منابع مالى \\ اين طرح با پشتيبانى مالى مركز تحقيقات بارورى و نابارورى صارم انجام \\ بذيرفت.
}

منابع

1. Alizadeh S, Hadizadeh M, Ameri H. Assessing the Effects of Infertility Treatment Drugs Using Clustering Algorithms and Data Mining Techniques. J-MazandUniv-Med-Sci [Internet]. 2014 Jul 1;24(114):26-35. Available from: http://jmums.mazums.ac.ir/article-13945-en.html

2. Fritz MA, Speroff L. Clinical Gynecologic Endocrinology and Infertility. 8th ed. Lippincott Williams \& Wilkins; 2011. 1488 p.

3. Peeraer K, Debrock S, De Loecker P, Tomassetti $\mathrm{C}$, Laenen A, Welkenhuysen M, et al. Low-dose human menopausal gonadotrophin versus clomiphene citrate in subfertile couples treated with intrauterine insemination: A randomized controlled trial. Hum Reprod. 2015 May 1;30(5):1079-88.

4. Seli E. Infertility [Internet]. 1st ed. Seli E, editor. Oxford, UK: Blackwell Publishing Ltd; 2011 [cited 2021 Apr 29]. 204 p. Available from: http://doi.wiley.com/10.1002/9781444393958

5. Dovey S, Sneeringer RM, Penzias AS. Clomiphene citrate and intrauterine insemination: analysis of more than 4100 cycles. Fertil Steril [Internet]. 2008
تصميم گيرى در انتخاب داروى مناسب كمك كند اما به عنوان يك شاخص، تاثير مستقل و مستقيمى بر انتخاب نوع بروتكل درمانى ندارند '1.]

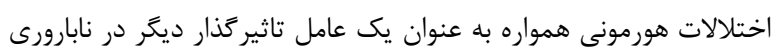

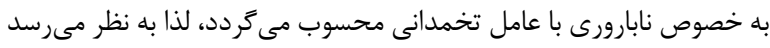

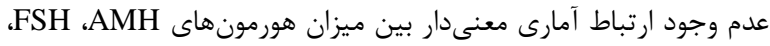

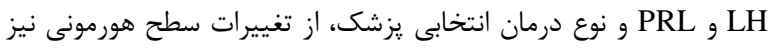

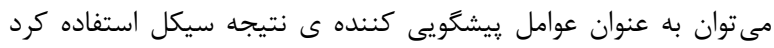

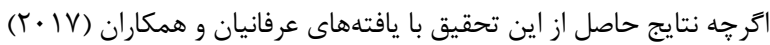

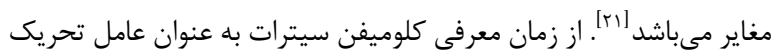
تخمك كذارى، هميشه بين ميزان تخمى كذارى و ميزان لقاح تخمك منها

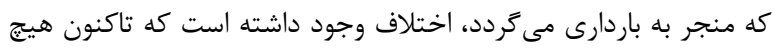

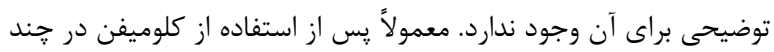

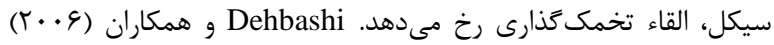

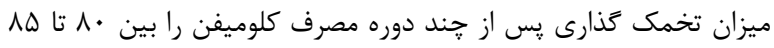

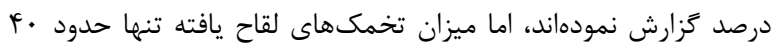

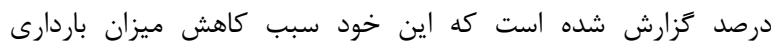

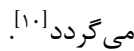
مقايسه نتايج حاصل از اين مطالعه بين دو يروتكل درمانى درونى نشان داد كه

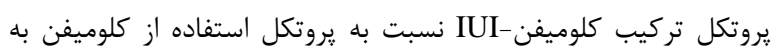

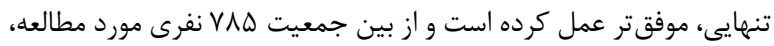

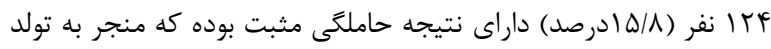

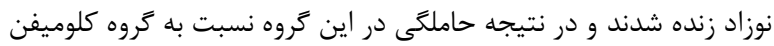

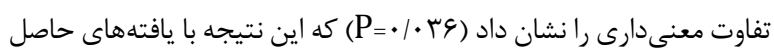

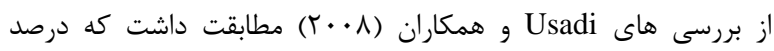

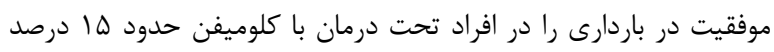
كزارش كرده بودند [بrا]

\section{نتيجه كيرى}

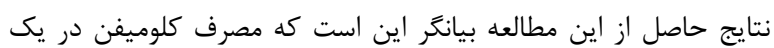

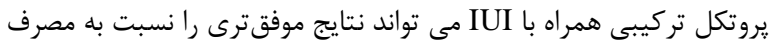

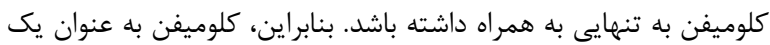

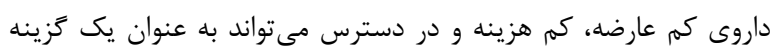

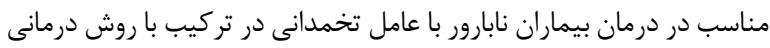

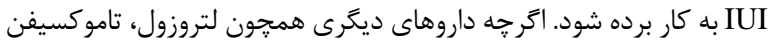

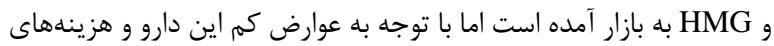

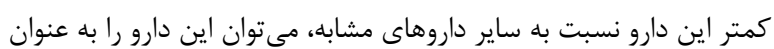

$$
\text { گزينه مناسبى براى درمان در نظر كرفت. }
$$

\section{ييشنهادات}

با توجه به اينكه كلوميفن سبب تحريك تخمك گذارى مى گردد، يُيشنهاد مىشود يك مطالعه RCT براى بررسى عوارض اين دارو در ايجاد تحريك تخميك 
endometrium and implantation. Yafteh [Internet]. 2011 [cited 2021 May 3];12(4):43-9. Available from: http://yafte.lums.ac.ir/article-1-330-en.html

17. Practice Committee of the American Society for Reproductive Medicine C. Use of clomiphene citrate in women. Fertil Steril [Internet]. 2006 Nov [cited 2021 May 3];86(5 SUPPL.). Available from: https://pubmed.ncbi.nlm.nih.gov/17055820/

18. Hassani Bafrani H, Abedzadeh M, Fruzanfard F, Tabasi Z. Effects of patient age, duration and cause of infertility and number of pre-ovulatory follicles on intrauterine insemination outcomes. Koomesh [Internet]. 2010 [cited 2021 May 3];12(1):59-65. Available from: https://koomeshjournal.semums.ac.ir/article-1-962en.html

19. Goswami PK, Khale A, Ogale S. Natural Remedies for Polycystic Ovarian Syndrome (PCOS) ?]: A Review [Internet]. Vol. 396, International Journal of Pharmaceutical and Phytopharmacological Research. [cited 2021 May 3]. Available from: www.eijppr.com 20. Dennett CC, Simon J. The role of polycystic ovary syndrome in reproductive and metabolic health: Overview and approaches for treatment. Diabetes Spectr [Internet]. 2015 May 1 [cited 2021 May 3];28(2):116-20. Available from: /pmc/articles/PMC4433074/

21. Erfanian Ahmad Pour M, Khalili Far H, Vakili Z. Comparison of pregnancy rates in levothyroxinetreated women with hypothyroidism with women without thyroid dysfunction disorders In patients referred to the infertility center for IVF in 2012 to 2013. Med Sci J Mashahad Azad Eslami Univ [Internet]. 2017 May 22 [cited 2021 May 3];7(1):118. Available from: http://medicine.sinaweb.net/article_531173.html

1.
Dec [cited 2021 May 3];90(6):2281-6. Available from: https://pubmed.ncbi.nlm.nih.gov/18191842/

6. The Practice Committee of the American Society for Reproductive Medicine C. Use of clomiphene citrate in infertile women: A committee opinion. Fertil Steril [Internet]. 2013 [cited 2021 May 3];100(2):341-8. Available from: https://pubmed.ncbi.nlm.nih.gov/23809505/

7. Gardner DK, Weissman A, Howles CM, Shoham Z. Textbook of Assisted Reproductive Techniques: Volume 2: Clinical Perspectives. 5th ed. CRC Press; 2018. $1040 \mathrm{p}$.

8. Tatem AJ, Beilan J, Kovac JR, Lipshultz LI. Management of anabolic steroid-induced infertility: Novel strategies for fertility maintenance and recovery. Vol. 38, World Journal of Men's Health. Korean Society for Sexual Medicine and Andrology; 2020. p. 141-50.

9. Dickey RP, Holtkamp DE. Development, pharmacology and clinical experience with clomiphene citrate [Internet]. Vol. 2, Human Reproduction Update. Hum Reprod Update; 1996 [cited 2021 May 3]. p. 483-506. Available from: https://pubmed.ncbi.nlm.nih.gov/9111183/

10. Dehbashi S, Vafaei H, Parsanezhad MD, Alborzi S. Time of initiation of clomiphene citrate and pregnancy rate in polycystic ovarian syndrome. Int J Gynecol Obstet [Internet]. 2006 [cited 2021 May 3];93(1):44-8. Available from: https://pubmed.ncbi.nlm.nih.gov/16530767/

11. Kistner RW, Smith OW. Observations on the use of a non-steroidal estrogen antagonist: MER-25. Surg Forum. 1960;10:725-9.

12. Greenblatt RB. Chemical induction of ovulation. Fertil Steril. 1961 Sep 1;12(5):402-4.

13. Usadi RS, Fritz MA. Induction of Ovulation with Clomiphene Citrate. Glob Libr Women's Med [Internet]. 2008 [cited 2021 May 3]; Available from: http://www.glowm.com/index.html?p=glowm.cml/s ection_view\&articleid=336

14. Seli E, Arici A. Ovulation induction with clomiphene citrate [Internet]. UpToDate. 2019. Available from: https://www.uptodate.com/contents/ovulationinduction-with-clomiphenecitrate\#: :text=Clomiphene was a revolutionary advance,polycystic ovary syndrome (PCOS).

15. Gardner DK, Simón C. Handbook of in vitro fertilization. CRC press; 2017.

16. Beigi Boroujeni M, Beigi Boroujeni N, Salehnia M, Beigi Boroujeni M. Ovarian stimulation, 